\title{
A Flat Organizational Structure for an Inclusive, Interdisciplinary, International, and Undergraduate-Led Team
}

\author{
Sriram Palepu, Alejandra Nitsch, Maitreyi Narayan, Stephany Kim and Nicole (Nico) Osier* \\ Osier Laboratory, College of Natural Sciences, McCombs School of Business, Dell Medical School and School of Nursing, \\ The University of Texas at Austin, Austin, TX, United States
}

OPEN ACCESS

Edited by:

Monica Byrne-Jimenez, Indiana University Bloomington,

United States

Reviewed by:

Paul R. Hernandez,

Texas A\&M University, United States

Marsha E. Modeste,

Pennsylvania State University (PSU),

United States

*Correspondence:

Nicole (Nico) Osier

nicoleosier@utexas.edu

Specialty section:

This article was submitted to

Leadership in Education,

a section of the journal

Frontiers in Education

Received: 24 August 2019 Accepted: 03 June 2020

Published: 14 July 2020

Citation:

Palepu S, Nitsch A, Narayan M, Kim S and Osier N (2020) A Flat

Organizational Structure for an

Inclusive, Interdisciplinary,

International, and Undergraduate-Led

Team. Front. Educ. 5:102.

doi: 10.3389/feduc.2020.00102
Here, we describe our team's approach to fostering an inclusive laboratory culture that executes a study in accordance with funder guidelines and research regulations, as well as provides volunteers with targeted mentorship, skill-development, and leadership opportunities. Our approach is five-pronged: (1) applying the principles of transformational leadership and kaizen, (2) establishing a strong mentor-mentee relationship and fostering community, (3) expanding the role of the research volunteer, (4) identifying volunteer leaders to (a) oversee and work alongside members of existing committees and (b) create new interconnected/interdisciplinary teams, and (5) using technology effectively. Our team is large; at the time of the initial submission we had 104 undergraduate/post-baccalaureate students with no graduate student supervision, and we have since grown to 118 members. Volunteers are diverse and come from 14 countries spanning four continents and numerous intersectional identities. We attribute our rapid expansion and diverse membership to our unique flat organization structure, which allows students from diverse backgrounds to work alongside the PI and contribute to improvements within the team, while fostering their own leadership skills. All volunteers are encouraged to take an active role in the lab, based on their strengths, experiences, and goals, and promote continuous improvement within the organization. Volunteers report feeling valued and have recruited new members from other groups they belong to, creating a self-sustaining system that allows aspiring professionals to learn from and lead their peers.

Keywords: flat organization, transformational leadership, student leadership, diversity, inclusivity, team culture

\section{TRANSFORMATIONAL LEADERSHIP AND THE KAIZEN PHILOSOPHY}

The two overarching principles that guide our team are those of transformational leadership and the kaizen philosophy; each will be briefly defined here and examples provided surrounding how they are applied within our team. Transformational leadership is a form of leadership in which leaders deeply trust and respect their team members, and work alongside them to accomplish objectives. Specifically, this leadership style is defined by (1) serving as a role model, (2) motivating and inspiring followers, (3) providing a range of ideas and solutions to roadblocks, and (4) mentoring individuals to unlock their full potential (Asif et al., 2019). Moreover, transformational leadership 
is distinguished by the fact that leaders advocate for the longterm needs of team members and embrace change; this is opposed to transactional leadership in which leaders satisfy the current needs of team members through give-and-take exchanges (Yammarino et al., 1993).

Working alongside a transformational leader, members are encouraged to share their experiences with the PI. These experiences may take the form of acknowledging inefficiencies and can transform into actionable improvement. By uncovering team perspectives on workflow processes, the PI is able to collaborate with the team to make incremental improvements through the kaizen philosophy. The kaizen philosophy was popularized by Japanese theorist Masaaki Imai, and posits that within an organization, continuous improvement can and should occur between all team members and in all areas of operation (García-Alcaraz et al., 2013). Evidence suggests the kaizen implementation increases management skills and participatory problem solving (Higuchi et al., 2015; von Thiele Schwarz et al., 2017).

Initially, we implemented the kaizen philosophy through meetings with a Vibe Tribe, a group of individuals tasked with encouraging "positive intergroup relations," characterized by members of both minority and majority groups having positive experiences working with each other (Ramarajan and Thomas, 2012). The Vibe Tribe provided a platform that directly targeted areas of improvement, in accordance with the Kaizen Philosophy, focusing team culture, and accountability. As our team expanded, and the various tasks became increasingly formalized, additional avenues for promoting the kaizen philosophy were pursued. Working groups were developed and met regularly with specific agendas, including revising SOPs, condensing training materials, and developing new resources (e.g., literature review template). New initiatives arose out of these working groups to address areas of improvement, such as instituting an application system and implementing a volunteer contract. The innovation arising from working groups made the value of developing regular kaizen meetings clear.

Regular kaizen meetings help to address the need for constant organizational improvement, and are where laboratory leaders and newer members work together to evaluate current practices and suggest areas of improvement. Kaizen meetings take place with various goals in mind including, but not limited to, forumstyle think tanks, topic oriented working groups, and meetings aimed at updating internal websites and training materials. The outcomes of these meetings vary, but some examples include creating several team calendars to increase accountability and minimize overlap in screening or consenting shifts; redefining roles and responsibilities for various positions within the laboratory; consolidating resources into one central access point within AirTable; and implementing Quick Response (QR) codes for meeting attendance. The kaizen philosophy remains the core foundation of the success of many strategies previously described and makes full use of the value of the flat organizational structure.

We have seen that the transformational leadership style and kaizen philosophy complement each other. Transformational leadership encourages team members to feel wholly committed to the lab and engaged in their work. Deep involvement encourages members to give honest feedback and suggestions, which are heeded to make constant improvements. In turn, implementation of member feedback encourages further involvement and satisfaction.

\section{ESTABLISHING A STRONG MENTOR-MENTEE RELATIONSHIP AND FOSTERING A COMMUNITY THAT VALUES DIVERSITY}

Prior to taking on their first volunteer, our principal investigator (PI) spent considerable time reflecting on their own past experiences as both a mentee and a mentor; this information was used to develop and refine their own mentorship strategy and tailor it to best meet individual member needs. New members complete an initial self-assessment and meet with our PI to set goals and establish rapport. The impact that closer, more personable mentorship had on them motivated the PI to use a similar approach on our team. Examples of strategies the PI uses to establish rapport include: encouraging all team members to refer to them by first name, sharing pronouns, as well as paying attention to, and providing recognition of birthdays, milestones, and other noteworthy accomplishments.

While our laboratory's PI does not come from a minoritized racial group, they are a first-generation college student and member of the lesbian, gay, bisexual, transgender (LGBT+) community. A key component of the initial rapport-building efforts is demonstrating a sincere commitment to an inclusive environment where all members are treated with dignity and respect. As part of the effort, the PI shares their own pronouns in the application materials and solicits volunteer pronouns via an online Google Form; in an effort to respect individual privacy "prefer not to answer" is included as an answer choice. Thus, far, no member has indicated they preferred not to share. All sensitive questions asked of volunteers are done with the promise of full confidentiality unless consent is provided to disclose this information; furthermore, whether this information is solicited using either a google form or other means, sensitive questions are explicitly optional. For example, one question allows students to identify as either cisgender heterosexual individuals, being somewhere on the LGBT+ spectrum, or preferring to NOT answer. Since evidence suggests that discrimination affects the career decisions of LGBT+ people, creating a safe and inclusive environment is imperative (Schneider and Dimito, 2010).

Notably, evidence has shown that a demographic match between the mentor and mentee is less important than shared values (Hernandez et al., 2017). Exploring personality and preferences, providing flexibility, communicating openly, and fostering trust are effective mentorship strategies that turn students into leaders (Hund et al., 2018). Onboarding resources include a map and statistics highlighting our team's diversity (see Figure 1). As our laboratory's culture of inclusivity and acceptance attracts diverse members, it has become a part of the lab's foundation to appreciate the differences that make each member unique. In an effort to promote this, all team members are expected to make a conscious effort to learn the correct 


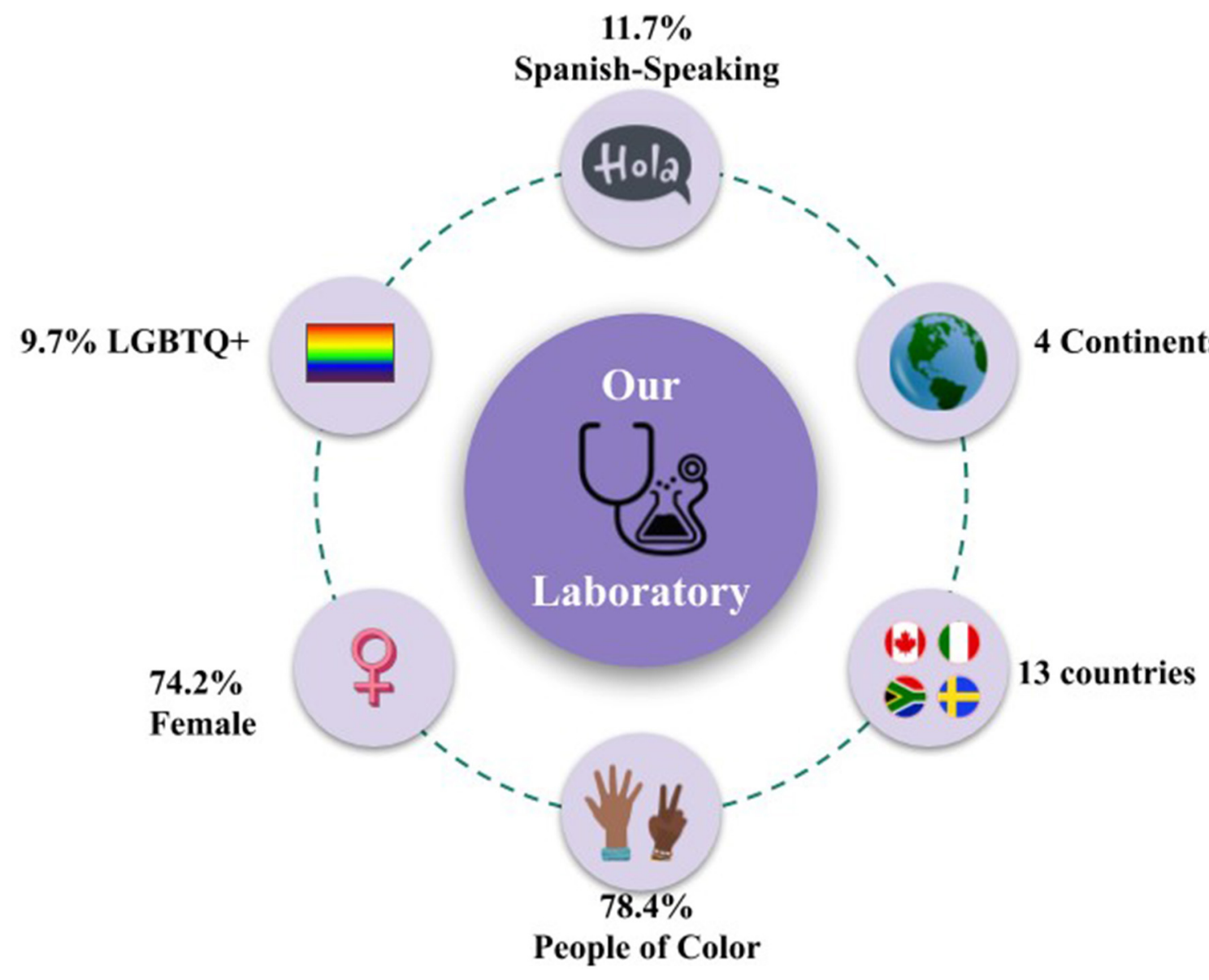

FIGURE 1 | Statistics representing the diversity of our team.

pronunciation of their teammate's names using the guide in the directory. Additionally, information shared at the initial meeting is used to identify opportunities for volunteers to get involved in the team while waiting on completion of mandatory background checks and regulatory approvals. For example, individuals who share a love of baking are encouraged to make baked goods which are delivered along with a monthly newsletter to be shared with the physicians, nurses, and other hospital staff at the recruitment site.

Starting from the initial interaction, the PI seeks to embody a transformational leadership style in which the leader works closely with the team to plan, execute, and troubleshoot initiatives. The PI participates, to whatever extent is feasible, in every aspect of the team's research operations, ranging from routine tasks such as de-icing freezers and assembling blood collection kits, to complex tasks such as maintaining regulatory compliance and enrolling participants. Consistent with the kaizen philosophy, the PI also regularly solicits feedback from all team members regarding how to improve their mentorship and research operations.

\section{EXPANDING ROLES OF UNDERGRADUATE AND POST-BACCALAUREATE VOLUNTEERS}

Volunteers are given the opportunity to expand their roles in the laboratory and contribute in non-traditional ways that are flexible and allow them to hone transferable skills. These expanded roles are based on individual hobbies and interests which are discussed during onboarding. From graphic design and videography, to creative writing and baking, each individual is encouraged to apply their passions and skills to kaizen some facet of the team's operations. To help ensure members feel valued for their contributions, the significance of these contributions is made clear. Assessing volunteers' self-perceived skillsets led to numerous innovations and improvements, including the development of committees. One example of a committee that arose organically, based on volunteer experience and interest, was the Public Relations Committee. This committee leads numerous initiatives including: managing social media accounts (Twitter, Instagram, Facebook, LinkedIn), designing recruitment flyers, 


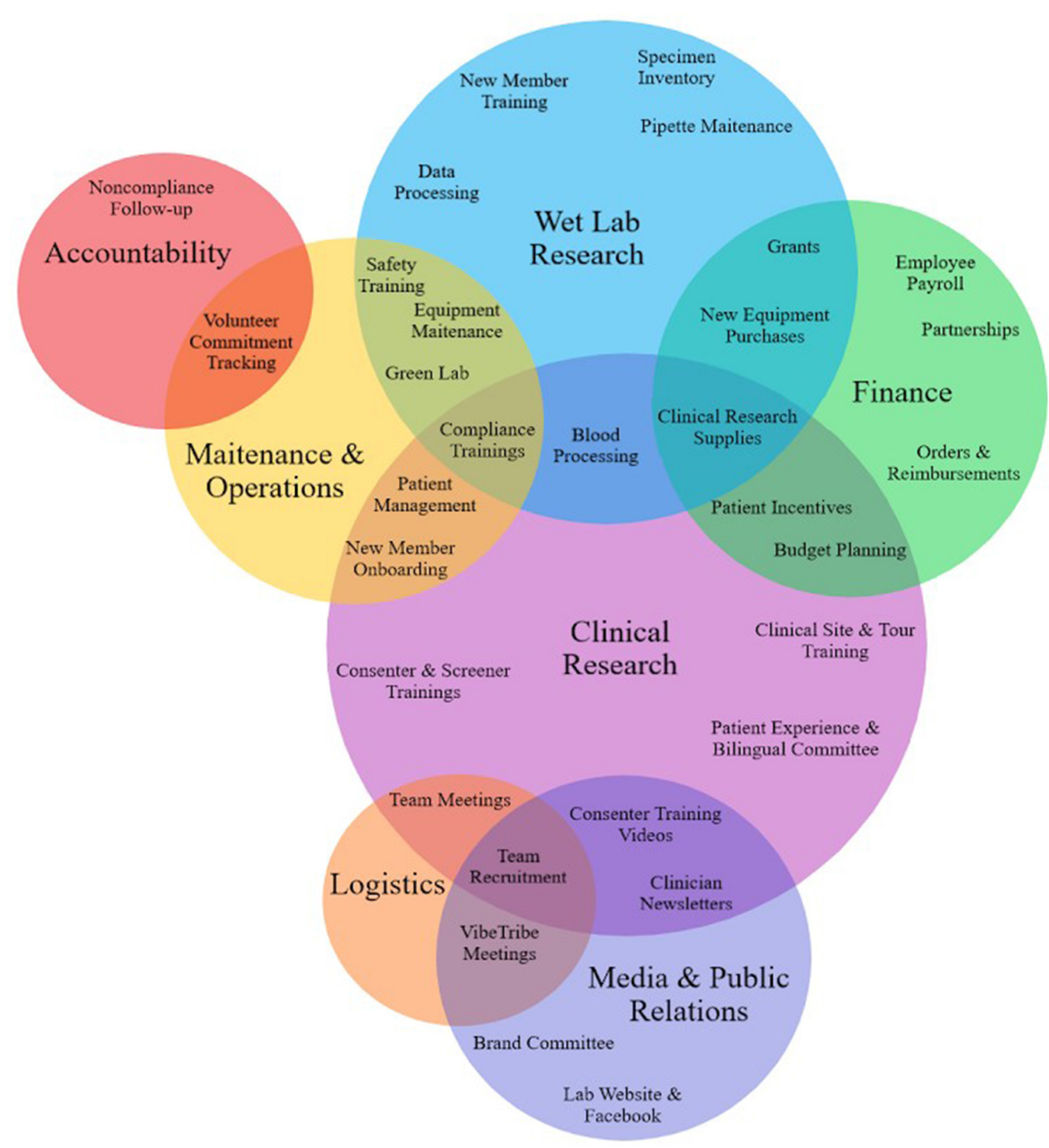

FIGURE 2 | Visual demonstrating the overlapping roles among the lab's cross-functional committees.

distributing a monthly newsletter to clinicians, and developing and updating the official team website.

Our lab recognizes and values proactivity and self-directed initiative. Newly onboarded members are reminded that existing leaders were self-appointed and encouraged to let the PI know when they are ready for a leadership role and what skills they bring to the table and/or hope to further hone. After identifying volunteers who show initiative and leadership skills, the PI gives them more responsibility via a role as a point of contact, trainer, and/or committee chair (see Figure 2).
While most laboratory teams consist of graduate students and a select few undergraduates, our team has 118 current active undergraduate and post-baccalaureate volunteers, most who plan on applying to graduate school. Having a large team with a variety of majors and skill sets allows for interdisciplinary collaboration and the capability to take on specialized, non-traditional roles. For example, finance students upkeep the lab expenditures and help with grant applications. Environmental studies students have helped the lab to be more sustainable, by earning UT Green Labs certification. Another initiative was instating an application 
system to holistically evaluate prospective members. The first application cycle was competitive, with 197 applicants for only 25 spots; the second application cycle received 146 applications and accepted 34 new members. Applicants frequently cite the culture of the laboratory and the depth and variety of opportunities as their motivation joining. Having a large team has facilitated significant improvements and advancements in a short amount of time. In addition to the initiatives described above, availability of personnel allowed us to recruit 85 diverse participants during the first year of the study. On average, each team member spends $7.89 \mathrm{~h}$ a week volunteering - more than $2 \mathrm{~h}$ greater than contractually required. The laboratory's culture and opportunities for leadership encourage members to work efficiently to make the most of their experiences.

\section{DEVELOPING LEADERSHIP SKILLS}

Our flat organizational structure contributes to our continued growth and success. The traditional, hierarchical organizational structure does not exist. In its place is a highly interconnected system that allows everyone, from the newest member to the $\mathrm{PI}$, to assume responsibility and contribute to incremental improvement within the organization by applying the kaizen philosophy. There are individuals who serve as points of contact for various tasks within the laboratory, but those individuals work closely with the PI and other volunteers. Every member is given the opportunity to become a leader and is provided with an arena to contribute their unique skills and talents. Laboratory leaders delegate easier tasks (e.g., data entry and inventorying) to newer members, while providing rationale, assistance, and gratitude. One way our PI encourages students to take initiative is through designing laboratory protocols and standard operating procedures (SOPs). When volunteers identify limitations in current SOPs, they take the initiative to revise them and share relevant updates with team members. It is imperative that the laboratory is able to communicate important procedures, to facilitate handoff of leadership positions upon volunteer matriculation. The flat organizational structure allows our laboratory to demonstrate "organizational resilience" by increasing cooperation, flexibility, and ability to act (Andersson et al., 2019).

In our efforts to be interdisciplinary and inclusive, we find that many of our laboratory members join from non-traditional backgrounds and with various talents and skills. Our team presently includes an ex-medical photographer, a physician seeking re-residency in the U.S., and a bartender. When members initially join, the aforementioned onboarding Google Form allows members to indicate demographic data, goals, and unique skills. We utilize this information opportunity to create new leadership roles. For example, in analyzing our data we noticed that many of the participants were Spanish speaking. The Latinx members of our team led a Spanish speaking committee to build trust and mitigate the language and cultural barriers (Sage et al., 2018). These members addressed the underrepresentation of Latinx individuals as both participants and researchers by translating enrollment paperwork and streamlining the communication process for non-Spanish speaking consenters. Allowing minority volunteers to lead is especially important; one study found that a research mentorship program at a Minority Serving Institution increased participation in advanced academic STEM training (Carpi et al., 2017) and professional STEM careers (Carpi et al., 2017; Estrada et al., 2018). Another volunteer started a committee focused participant payments and following up when surveys are overdue to reduce attrition. Upon realization of the difficulty in recruiting and retaining participants, several volunteers used their talents to create an official laboratory logo, postcards, and monthly newsletters that allowed the laboratory to gain more attention and awareness at the clinical recruitment site.Volunteers have full control over their own level responsibility they want to take on, thus enabling them to become active members and leaders.

It is crucial that we have accountability protocols to ensure that every member is working during their designated shifts to maximize patient recruitment. When on a screening or consenting shift, volunteers must be in communication not only to alert one another when there is a potential case, but also to support one another during the consenting process. In this way, volunteers are kept accountable for shifts they sign up for. Per laboratory policy, members are allowed up to three exemptions and two strikes for days when they are feeling under the weather or have an emergency; thus over a typical 16 week semester, members are expected to fulfill expectations for at least 11 weeks. This accountability system encourages members to be responsible.

Ultimately, our team develops transformational leaders. Laboratory leaders work alongside other members to take care of administrative duties, maintain operations, and launch new initiatives. Through leading by example, our leaders show the importance of encouraging and enabling members of the team. With the plethora of opportunities available to lead and learn from others, our members acquire leadership skills that will prepare them for their roles as future professionals.

\section{TECHNOLOGY}

Several technological applications are used to (1) facilitate individual communication, (2) manage team productivity, and (3) provide opportunities to participate remotely. Opportunities for remote engagement have benefitted team members who have pursued study abroad opportunities or graduated but wanted to maintain involvement. Technology allows our PI and other team leaders to communicate with all members en masse, which aligns with evidence indicating that using communication technology cuts costs, improves productivity, and saves time (Arvanitis and Loukis, 2009). Several free applications are used by our team for various purposes. Each will be briefly described below; please note that alternatives are available and the authors report no conflict of interest.

\section{Slack}

Slack is a business communication platform that allows teams to communicate through channels-collaboration hubs where people can share documents, make announcements, set 
reminders, and poll team members. The platform can be accessed through a mobile app or the Slack website. While email is used for formal communication, Slack allows volunteers to clarify their doubts with teammates through group messages and message boards; individuals that need the PI's assistance can tag them directly. Many working in academia receive hundreds of emails a day, which contributes to "email stress," the fatigue associated with high email volume (Jerejian et al., 2013). Taking away the reliance on email is crucial in allowing our PI to manage such a large team effectively, while preserving time for higher-level functions. Moreover, volunteers receive shoutouts for their work on the Slack platform in a dedicated channel (\#KaizenKweens), which serves to celebrate team accomplishments and keep members engaged. While several communication platforms exist that serve a similar purpose (e.g., GroupMe, Fuze, Workzone), the ultimate purpose is to streamline communication to allow leaders to respond and reach out to team members in a clear, timely, and direct manner.

\section{Google Forms}

Google Forms is a surveying application that automatically compiles responses into a spreadsheet. Our team uses Google Forms for several recordkeeping purposes in the lab, including accountability, onboarding, and clinical engagement. Productivity is managed through a weekly Google Form, created and sent out by the chair of the Accountability Committee. All members fill out the form with hours worked per week, action items for other committee members, and updates/requests for our PI. This system allows for monitoring contract adherence and conveying pressing messages to our PI, with minimal oversight. Most importantly, these surveys play a significant role in adhering to the kaizen philosophy by allowing members to submit feedback and ideas. Ideas that have arisen from survey feedback include developing a form to improve reference letter writing and hosting a resume workshop. Furthermore, all students who screen and consent patients fill out a Google Form after their shift, which is tracked by the Chief Clinical Officer and Data Management Committee as a source of data for future presentations and publications, and to ensure regulatory compliance.

\section{AirTable}

One of the primary challenges in effectively leading large teams is task delegation, which we accomplish through AirTable. This database platform not only serves to itemize the tasks for each committee but is also where volunteers can assign themselves to projects directly posted by the PI. The types of tasks range significantly: from improving social media presence to writing SOPs for grants and publications. These projects allow volunteers to choose their degree of involvement in the lab, beyond the minimum requirements. Not only does this allow members to take leadership in the tasks they are interested in, but AirTable provides clear documentation of individual contributions, which is referenced when nominating students for awards and writing letters of recommendation.

In summary, our team is able to maintain its size because the team embraces technology and continually seeks better ways to use technology as consistent with the kaizen philosophy. Feedback, mentorship, and group communication are facilitated by several apps and tools that allow the PI to practice transformational leadership, by working alongside and directly communicating with team members. Members can access a support network of mentors because everyone is readily accessible on several platforms. Use of electronic collaboration tools allows members to easily provide feedback, update the team regarding their progress, and share barriers that need to be addressed in adherence to the kaizen philosophy.

\section{CONCLUSION}

The two overarching principles that guide our team are (1) transformational leadership and (2) the kaizen philosophy. These interconnected principles have allowed our team to grow into a diverse, inclusive, and productive community. Our flat organizational structure provides direct benefits for both volunteers and the PI. Working alongside the PI provides members with direct mentorship and feedback, while fostering a sense of community. Hearing the experiences of members allows the PI to identify targeted areas for improvements and work with members to apply their skill sets to develop new initiatives. We hope the strategies and methods outlined in this manuscript are useful in helping other PIs and mentors manage and lead their teams more effectively.

\section{AUTHOR CONTRIBUTIONS}

SP wrote the first draft of the abstract and outlined the manuscript. Each of the five sections was expanded upon by all the authors. Establishing a strong mentor-mentee relationship and fostering a community that values diversity was written by NO. Expanding Role of Undergrad Student was written by MN. Developing Leadership Skills was written by SK. Technology was written by SP, and The Kaizen Philosophy was written by AN. All authors provided edits and revisions for the other sections. Additionally, AN, NO, and $\mathrm{MN}$ created the two figures for the manuscript.

\section{FUNDING}

This study was generously supported by the St. David's Center for Health Promotion and Disease Research (CHPR) Pilot Grant and the Heilbrunn Family Center for Nursing Research Grant. Additional financial support for this project came from the Rising STARs Award from the University of Texas System Permanent University Fund Bond, which was awarded to Dr. Osier.

\section{ACKNOWLEDGMENTS}

We would like to thank the Dell Children's Medical Center for allowing us to conduct our study at their facilities, and Kimberly 
Lewis for helping us obtain site approval. Additionally, we would like to thank the patients and their families who chose to join the study. Lastly, we give our thanks to the members of the Osier

\section{REFERENCES}

Andersson, T., Cäker, M., Tengblad, S., and Wickelgren, M. (2019). Building traits for organizational resilience through balancing organizational structures. Scand. J. Manag. 35, 36-45. doi: 10.1016/j.scaman.2019.01.001

Arvanitis, S., and Loukis, E. N. (2009). Information and communication technologies, human capital, workplace organization and labour productivity: a comparative study based on firm-level data for Greece and Switzerland. Inf. Econ. Policy. 21, 43-61. doi: 10.1016/j.infoecopol.2008.09.002

Asif, M., Jameel, A., Hussain, A., Hwang, J., and Sahito, N. (2019). Linking transformational leadership with nurse-assessed adverse patient outcomes and the quality of care: assessing the role of job satisfaction and structural empowerment. Int. J. Environ. Res. Public Health. 16:2381. doi: 10.3390/ijerph16132381

Carpi, A., Ronan, D. M., Falconer, H. M., and Lents, N. H. (2017). Cultivating minority scientists: undergraduate research increases self-efficacy and career ambitions for underrepresented students in STEM. J. Res. Sci. Teach. 54, 169-194. doi: 10.1002/tea.21341

Estrada, M., Hernandez, P. R., and Schultz, P. W. (2018). A longitudinal study of how quality mentorship and research experience integrate underrepresented minorities into STEM careers. CBE Life Sci. Educ. 17:ar9. doi: 10.1187/cbe.17-04-0066

García-Alcaraz, J. L., Rivera, D. G., and Alvarado-Iniesta, A. (2013). Critical success factors for Kaizen implementation in manufacturing industries in Mexico. Int. J. Adv. Manufact. Technol. 68. doi: 10.1007/s00170-0134750-2

Hernandez, P. R., Estrada, M., Woodcock, A., and Schultz, P. W. (2017). Protégé perceptions of high mentorship quality depend on shared values more than on demographic match. J. Exp. Educ. 85, 450-468. doi: 10.1080/00220973.2016.1246405

Higuchi, Y., Nam, V. H., and Sonobe, T. (2015). Sustained impacts of Kaizen training. J. Econ. Behav. Org. 120, 189-206. doi: 10.1016/j.jebo.2015.10.009

Hund, A. K., Churchill, A. C., Faist, A. M., Havrilla, C. A., Love Stowell, S. M., McCreery, H. F., et al. (2018). Transforming mentorship in STEM by
Laboratory team, Linda Wasson who recommended volunteers for our team, and Joe Zaghrini who provided consultations on team management and accountability. training scientists to be better leaders. Ecol. Evol. 8, 9962-9974. doi: 10.1002/ ece 3.4527

Jerejian, A. C. M., Reid, C., and Rees, C. S. (2013). The contribution of email volume, email management strategies and propensity to worry in predicting email stress among academics. Comput. Human Behav. 29, 991-996. doi: 10.1016/j.chb.2012.12.037

Ramarajan, L., and Thomas, D. (2012). "A positive approach to studying diversity in organizations," in The Oxford Handbook of Positive Organizational Scholarship, eds K. S. Cameron and G. M. Spreitzer (Oxford: Oxford University Press). doi: 10.1093/oxfordhb/9780199734610.013.0041

Sage, R., Benavides-Vaello, S., Flores, E., LaValley, S., and Martyak, P. (2018). Strategies for conducting health research with Latinos during times of political incivility. Nurs. Open. 5, 261-266. doi: 10.1002/nop2.166

Schneider, M. S., and Dimito, A. (2010). Factors influencing the career and academic choices of lesbian, gay, bisexual, and transgender people. J. Homosex. 57, 1355-1369. doi: 10.1080/00918369.2010.517080

von Thiele Schwarz, U., Nielsen, k. M., Stenfors-Hayes, T., and Hasson, H. (2017). Using Kaizen to improve employee well-being: results from two organizational intervention studies. Hum. Relat. 70, 966-993. doi: 10.1177/0018726716677071

Yammarino, F. J., William, D. S., and Bass, B. M. (1993). Transformational Leadership and Performance: A Longitudinal Investigation. Leadersh. Q. 4, 81-102. doi: 10.1016/1048-9843(93)90005-E

Conflict of Interest: The authors declare that the research was conducted in the absence of any commercial or financial relationships that could be construed as a potential conflict of interest.

Copyright (c) 2020 Palepu, Nitsch, Narayan, Kim and Osier. This is an open-access article distributed under the terms of the Creative Commons Attribution License (CC $B Y)$. The use, distribution or reproduction in other forums is permitted, provided the original author(s) and the copyright owner(s) are credited and that the original publication in this journal is cited, in accordance with accepted academic practice. No use, distribution or reproduction is permitted which does not comply with these terms. 